\title{
Expression of Inorganic Pyrophosphatase (PPA1) Correlates with Poor Prognosis of Epithelial Ovarian Cancer
}

\author{
Hui Li, ${ }^{1}$ Ning Xiao, ${ }^{1}$ Zongpeng $\mathrm{Li}^{1}$ and Qin Wang ${ }^{1}$ \\ ${ }^{1}$ Nursing Department, Linyi People’s Hospital, Linyi, Shandong, China
}

Ovarian serous carcinoma (OSC) is the most common epithelial ovarian cancer. Inorganic pyrophosphatase (PPA1) catalyzes the hydrolysis of pyrophosphate to inorganic phosphate, thereby providing extra energy for metabolism. The significance of PPA1 in the prognosis of OSC has not been investigated. Our study aimed to explore the expression and predictive role of PPA1 in OSC progression. We screened the expression of PPA1 protein in OSC tissues from 139 patients by immunohistochemistry, and evaluated its correlation with clinicopathological characteristics. PPA1 was categorized as high expression in 58 OSC cases (41.7\%), which was correlated with poor differentiation, positive lymph node (LN) metastasis and advanced FIGO (The International Federation of Gynecology and Obstetrics) stages. Univariate and multivariate analyses identified PPA1 as a novel independent prognostic biomarker in OSC patients; meanwhile, conventional factors such as LN status and FIGO stages also showed statistical significance. Moreover, the expression levels of PPA1 protein were higher in A2780 and OVCAR3 human ovarian cancer cell lines than those in normal ovarian surface epithelial cells. Using these ovarian cancer cell lines, we showed that PPA1 overexpression caused the decrease in the expression level of p53, the tumor suppressor, with the increase in $\beta$-catenin level, as determined by Western blot analysis. Conversely, knockdown of PPAl expression was associated with the increase of p53 level and the decreased of $\beta$-catenin level. Consistently, the proliferation and invasion capacities of ovarian cancer cells were enhanced upon PPA1 overexpression. In conclusion, PPA1 serves as a potential prognostic biomarker for patients with OSC.

Keywords: biomarker; ovarian serous cancer; PPA1; prognosis; proliferation Tohoku J. Exp. Med., 2017 February, 241 (2), 165-173. C 2017 Tohoku University Medical Press

\section{Introduction}

Epithelial ovarian cancer (EOC) represents the second most common gynecological malignancy followed by cervical cancer, and surgery is the only curative therapy. Over half of female deaths caused by EOC are due to difficulties for early diagnosis and high recurrence rate (Vang et al. 2009; Siegel et al. 2016). The 5-year overall survival rate is less than $30 \%$ for the EOC patients with advanced stages (International Federation of Gynecology and Obstetrics [FIGO] stage III/IV), which shows limited improvement over the past decades (Cheon et al. 2014; Terry et al. 2016). Ovarian serous carcinoma (OSC) is the most common EOC type, with the proportion as high as $80 \%$ (Jayson et al. 2014). Developing effective therapies and predicting postoperative clinical outcomes are still the hot-topics in the study of OSC. Here we tried to explore novel biomarkers in OSC and hopefully contribute to a better understanding for the molecular mechanisms.

It has been determined that not only the dysregulation of molecule expression levels but also the post-translational modifications were involved in oncogenesis. Consequently, various protein enzymes are drawing more and more attentions, including kinases (Jung et al. 2016), phosphatases (Rajadurai and Havrylov 2016), ubiquitin ligases (Signoretti et al. 2016) and deubiquitinases (Liu et al. 2016). However, either the process of protein expression or modification needs energy; thus, energy dysregulation plays important roles in carcinogenesis and cancer progression. Inorganic pyrophosphatase (PPA1) is an enzyme that can hydrolyze pyrophosphate to phosphate; therefore, it functions as an extra energy source besides ATP (Yi et al. 2012). Proteomic studies have revealed the up-regulated expression of PPA1 in various malignancies compared with normal tissues (Chen et al. 2002; Tomonaga et al. 2004; Megger et al. 2013; Bodnar et al. 2016). In addition, PPA1 has been reported to participate in cancer related metabolic alterations in gastric cancer and serves as a negative prognostic marker (Yang et al. 2015). Most recently, the expression of PPA1 in EOC was also examined, which showed statistical

Received December 5, 2016; revised and accepted January 28, 2017. Published online February 16, 2017; doi: 10.1620/tjem.241.165. Correspondence: Qin Wang, Nursing Department, Linyi People's Hospital, 28 Jiefang Road, Linyi, Shandong 276000, China.

e-mail: wangqin_wqwq@126.com 
correlation with histological differentiation (Luo et al. 2016). However, it still remains to be investigated whether PPA1 expression can help to predict the clinical outcomes of EOS.

In the current study, we systematically explored the expression profiles of PPA1 mRNA and protein in OSC tissues, as well as its correlations with clinicopathological characteristics. Univariate and multivariate analyses were carried out to verify the significance of PPA1 in predicting overall survival for OSC patients, which further provided evidence for its potential value in chemotherapy development. We also investigated the possible downstream effectors of PPA1 in OSC by performing overexpression and knockdown assays in ovarian cancer cells.

\section{Methods}

\section{Patients and samples}

Informed consent for the analysis of tissue specimens in this study was collected from every patient. This research was approved by the Ethics Committee of Linyi People's Hospital. Fresh resected tissues from OSC patients were obtained from Department of Gynecology and Obstetrics during primary surgery, and were immediately frozen with liquid nitrogen and stored at $-80^{\circ} \mathrm{C}$ until use. Totally twelve fresh-frozen OSC tissues and paired adjacent nontumorous tissues were collected. Another 139 paraffin-embedded OSC tissues were obtained from the Department of Pathology during the period from January 2005 to January 2015 . The tumor stage and grade was classified according to the FIGO criteria (Cho and Shih Ie 2009).

For the 139 patients enrolled in our retrospective study, the median age was 53.3 years (range 22-87 years), and 50 patients
(36.0\%) were older than 60 years old. Thirty-four patients were histologically graded as well differentiated (G1), while 73 and 32 cases with moderately (G2) and poorly differentiated (G3), respectively. The cohort comprised 44 cases $(31.7 \%)$ with FIGO stage I/II, and the other 95 cases $(68.3 \%)$ with FIGO stage III/IV. Seventy cases $(50.4 \%)$ were identified with positive lymph node (LN) metastasis, and the other 69 cases (49.6) as LN-negative at the time of primary surgery. The clinicopathological features of enrolled patients are summarized in Table 1.

\section{Immunohistochemistry (IHC) and IHC evaluation}

Paraffin-embedded 3- $\mu \mathrm{m}$ sections from OSC tissues were used for IHC as describe previously (Chen et al. 2016). Sections were deparaffinized with dimethylbenzene and rehydrated through $100 \%$, $95 \%, 90 \%, 80 \%$ and $70 \%$ ethanol. The tissue slides were then boiled for $10 \mathrm{~min}$ by microwave for antigen retrieve, followed by treated with $3 \%$ hydrogen peroxide to eliminate endogenous peroxidase activity. Then the sections were blocked with $5 \%$ bovine serum albu$\min (\mathrm{BSA})$ for $1 \mathrm{~h}$ and later incubated with rabbit anti-PPA1 (1:1000 dilution; ab96099, Abcam, USA) at $4{ }^{\circ} \mathrm{C}$ overnight. BSA incubation was used as a negative control. After incubation with horseradish peroxidase-conjunctive second antibody for another $30 \mathrm{~min}$ at $37^{\circ} \mathrm{C}$, the slides were finally stained with DAB solution for visualization.

The expression levels of PPA1 were assessed based on the degree of staining and percentage of positive stained cells by two pathologists independently. No staining, light yellow, dark yellow, and brown were scored as 1,2,3, and 4, respectively. The mean percentage of positive stained cells were scored as followed: 1 for less than $25 \%, 2$ for $25-50 \%, 3$ for $50-75 \%, 4$ for $75-100 \%$. We calculated the final IHC score by add these two scores above: $0-5$ were considered as low expression, while 6-8 were considered as high expression.

Table 1. Clinical characteristics of OSC patients.

\begin{tabular}{|c|c|c|c|c|}
\hline \multirow[t]{2}{*}{ Variables } & \multirow{2}{*}{$\begin{array}{c}\text { Cases } \\
(n=139)\end{array}$} & \multicolumn{2}{|c|}{ PPA1 expression level } & \multirow[t]{2}{*}{ P value } \\
\hline & & Low $(n=81)$ & High $(n=58)$ & \\
\hline Age (years) & & & & 0.261 \\
\hline$\leq 60$ & 89 & 55 & 34 & \\
\hline$>60$ & 50 & 26 & 24 & \\
\hline Ascites (mL) & & & & 0.461 \\
\hline None or $\leq 1000$ & 77 & 47 & 30 & \\
\hline$>1000$ & 62 & 34 & 28 & \\
\hline Distribution & & & & 0.242 \\
\hline Unilateral & 103 & 63 & 40 & \\
\hline Bilateral & 36 & 18 & 18 & \\
\hline Pathological grade & & & & $<0.001 *$ \\
\hline G1 & 34 & 28 & 6 & \\
\hline G2 & 73 & 42 & 31 & \\
\hline G3 & 32 & 11 & 21 & \\
\hline LN metastasis & & & & $0.007 *$ \\
\hline Negative & 69 & 48 & 21 & \\
\hline Positive & 70 & 33 & 37 & \\
\hline FIGO stage & & & & $0.019^{*}$ \\
\hline $\mathrm{I} \sim \mathrm{II}$ & 44 & 32 & 12 & \\
\hline III $\sim \mathrm{IV}$ & 95 & 49 & 46 & \\
\hline
\end{tabular}

LN, lymph node; FIGO, International Federation of Gynecology and Obstetrics. 


\section{$R T-q P C R$}

Total RNA was extracted with TRIzol regent (Life Technologies, CA, USA) following the manufacturer's instructions. The concentration and quality of RNA was determined by spectrophotometry K5600. cDNA was then synthesized from $1 \mu \mathrm{g}$ of total RNA with High-Capacity cDNA Reverse Transcription Kit (Thermo Fisher Scientific, PA, USA). The mRNA expression levels of PPA1 and glyceraldehyde-3-phosphate dehydrogenase (GAPDH) were measured by real-time PCR system. The data were obtained by normalizing PPA1 cDNA cycle threshold $(\mathrm{Ct})$ values with corresponding GAPDH Ct, and then analyzed with $2^{-\triangle 4 C t}$ method.

The primers for human PPA1 cDNA was ordered from Thermo Fisher Scientific (Hs01067932_g1). The forward primer sequence for GAPDH cDNA was 5'-CCCACTCCTCCACCTTTGAC-3' and the reverse primer sequence was 5'-GGATCTCGCTCCTGGAAG ATG-3'.

\section{Cell culture and transfection}

IOSE-386 (OSE), a normal ovarian epithelial cell line, was obtained from the China Center for Type Culture Collection (CCTCC, Shanghai, China); serous ovarian carcinoma (SOC) cell lines (A2780 and OVCAR3) were purchased from American Type Culture Collection (ATCC, USA). All cells were grown in Dulbecco's Modified Eagle's Medium (DMEM) with $10 \%$ fetal bovine serum (FBS) at $37^{\circ} \mathrm{C}, 5 \% \mathrm{CO}_{2}$, with $100 \mathrm{IU} / \mathrm{mL}$ penicillin and $100 \mu \mathrm{g} / \mathrm{mL}$ streptomycin.

Human PPA1 cDNA was isolated using RT-PCR and subcloned into pcDNA vector (\#V79020, Thermo Fisher Scientific, PA, USA) to generate the pcDNA-PPA1 construct, which was later used for overexpression transfection with empty vector pcDNA as control. Small interfering RNA (siRNA) for PPA1 (\#105886) and negative control siRNA (ncRNA) were also ordered from Thermo Fisher Scientific. Briefly, A2780 and OVCAR3 cells were seeded into six-well plates, and then the cells were transfected with each plasmid or siRNA by Lipofectamine 3000 (Invitrogen, PA, USA) following the manufacturer's instructions. After transfection, cells were incubated for another $48 \mathrm{~h}$ and subjected to Western blot analyses.

\section{Western blot analysis}

Cells were collected and homogenized by RIPA lysis buffer, total protein concentration was measured with BCA protein assay kit. The proteins were electrophoresed by $10 \%$ SDS- polyacrylamide gel electrophoresis and then transferred to a polyvinylidene fluoride (PVDF) membrane, followed by incubation with 5\% BSA at room temperature for $1 \mathrm{~h}$. Rabbit anti-human PPA1 antibody (ab96099, Abcam, USA), moue anti-human $\beta$-actin antibody (sc-58673, Santa Cruz, CA, USA), rabbit anti-human $\beta$-catenin antibody (\#9562, Cell Signaling Technology, MA, USA), and moue anti-human p53 antibody (sc-47698, Santa Cruz, CA, USA), were incubated with the PVDF membranes at $4^{\circ} \mathrm{C}$ overnight, respectively. Secondary antibodies were then added and incubated at room temperature for $1 \mathrm{~h}$, followed by adding electrogenerated chemiluminescent (ECL) and exposed to X-ray films as reported by others (Pan et al. 2013). Each experiment was repeated for at least three times.

\section{MTT assay}

The effect of PPA1 on cell viability was determined by MTT assay using tetrazolium 3-[4,5]-dimethylthiahiazo(-z-y1)-3,5-di-phe- nytetrazoliumromide dye (Guo et al. 2015). Briefly, transfected cells were seeded into a 96 -well plate $\left(4 \times 10^{3} /\right.$ well $)$ and allowed for adhesion overnight. At designated times (0, 24, 48, 72, $96 \mathrm{~h}), 20 \mu \mathrm{L}$ of MTT solution was added into each well, and the cells were incubated for another $4 \mathrm{~h}$ at $37^{\circ} \mathrm{C}$. The solution was then removed, and $150 \mu \mathrm{L}$ dimethyl sulfoxide (DMSO) was added to each well. The absorbance of each sample was measured at $570 \mathrm{~nm}$ using a microplate reader. Each experiment was repeated for at least three times.

\section{Transwell assay}

Invasion assay was determined using Matrigel invasion chambers (BD Bioscience, CA, USA). A2780 and OVCAR3 cells were transfected and cultured for another 24 hours; then cells were re-suspended and seeded in the upper chambers $\left(1 \times 10^{5}\right.$ cell/well $)$ and cultured for $8 \mathrm{~h}$ to allow for adhesion. The medium in the upper chambers was changed to serum-free DMEM, while the bottom chamber was filled with DMEM containing 10\% FBS. After incubation for further $24 \mathrm{~h}$, the upper surfaces of the Transwell chambers were scraped with cotton swabs, and the migrated and invaded cells were fixed with $4 \%$ paraformaldehyde, and then stained with Giemsa solution. The stained cells were photographed and counted under a light microscope from five randomly selected fields. Each experiment was repeated for at least three times.

\section{Statistics}

Each patient had completed follow-up information. Survival time was defined from the day of the first operation to death or last day of follow-up (February 1st, 2016). Statistical evaluation of the data from PCR and cell experiments were analyzed using $t$ test. Chisquare $\left(\chi_{2}\right)$ tests were performed to evaluate whether PPA1 expression was correlated with clinicopathological parameters. Patients' overall survival curves were plotted by the Kaplan-Meier method and subjected to log-rank test, with factors including age, ascites, tumor distribution, pathological grade, LN metastasis and FIGO stage. Factors shown to be of prognostic significance in univariate models were analyzed in a multivariate Cox regression model to further evaluate their independent prognostic values. The hazard ratio (HR) was calculated by setting the group with the lowest hazard as reference group. For all analyses, a P-value $<0.05$ was regarded as statistically significant. Data were analyzed using SPSS 19.0 statistics software (SPSS, USA).

\section{Results}

PPA1 expression profiles in OSC and its correlation with clinicopathological characteristics

PPA1 showed higher expression in OSC tissues than that in normal ovarian tissues, which presented immunostaining in the cytoplasm of tumor cells (Fig. 1A, B). Among the 139 cases, 58 patients $(41.7 \%)$ were identified with high PPA1 expression (Table 1), while the other 81 cases $(58.3 \%)$ were grouped as low PPA1 expression (Fig. 1C). It is noteworthy that the expression levels of PPA1 mRNA were also significantly higher in tumor tissues than those in matched adjacent non-tumorous tissues (Fig. 1C). Details of the correlation between PPA1 protein expression levels and clinicopathological characteristics of OSC are shown in Table 1. IHC results showed that PPA1 protein level was higher in stage III/IV patients than that in patients 

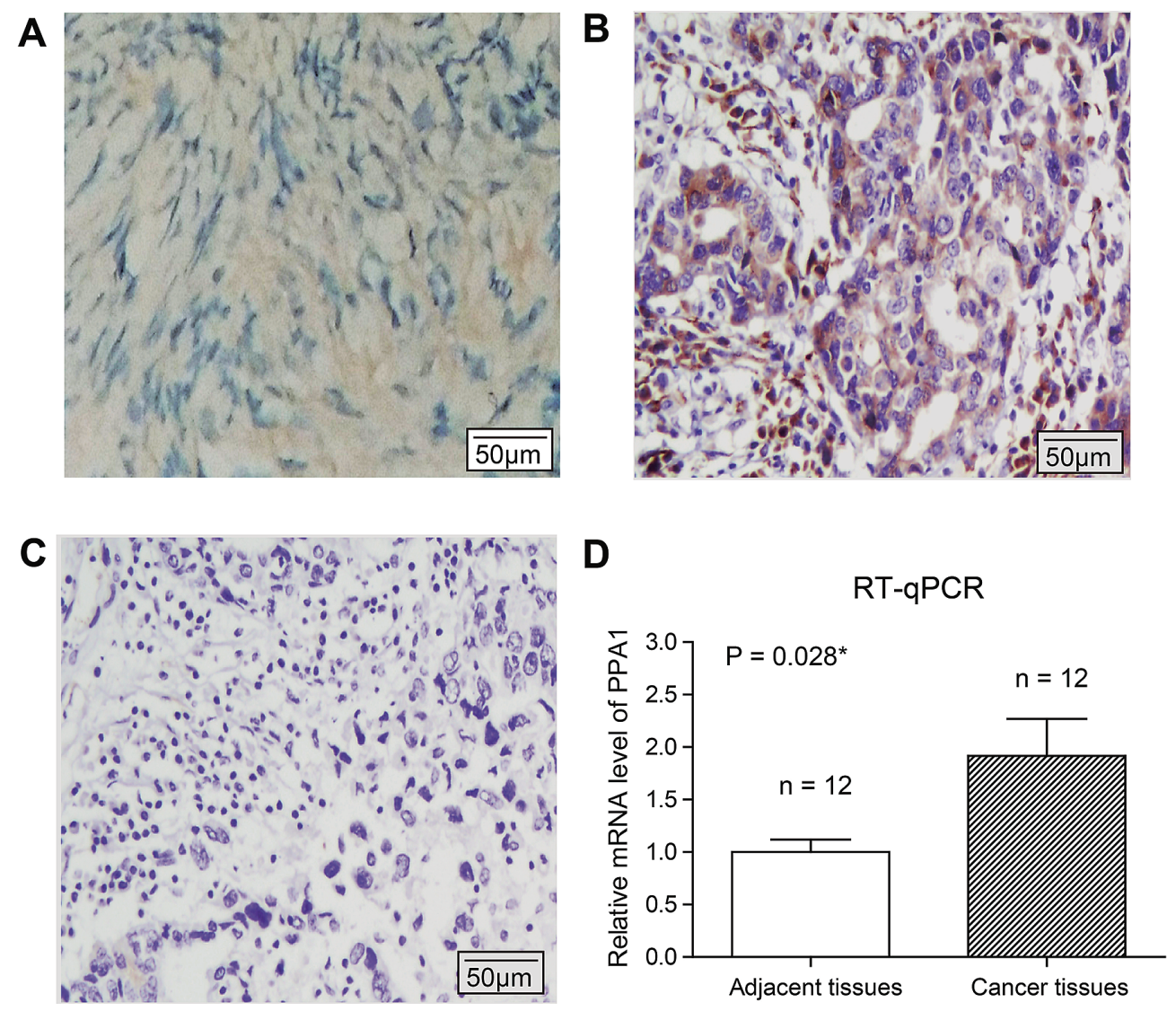

Fig. 1. Expression of PPA1 in ovarian serous cancer tissues.

(A) PPA1 was negatively expressed in normal ovarian tissues. (B) Representative high expression of PPA1 in the ovarian serous cancer tissue. The score of positive cell percentage is 4 and the score of staining intensity is 3 ; thus, the final IHC score is 7 (calculated by score of positive cell percentage plus score of staining intensity) and defined as PPA1 high-expression. (C) Representative low expression of PPA1 in the ovarian serous cancer tissue. The score of positive cell percentage is 1 and the score of staining intensity is 1 . Total score is 2, thus defined as PPA1 low expression. (D) RT-qPCR results of PPA1 mRNA levels in ovarian cancer tissues and adjacent non-tumorous tissues. The level of PPA1 mRNA in cancer tissues is 1.92 -fold higher than that in adjacent tissues $(\mathrm{n}=12, \mathrm{P}=0.028)$. Scale bar: $50 \mu \mathrm{m}$.

with stage I/II $(\mathrm{P}=0.019)$. High PPA1 protein expression was also significantly associated with positive LN metastasis $(\mathrm{P}=0.007)$. Moreover, the chi-square analysis demonstrated that the PPA1 expression was significantly correlated with pathological differentiation grade $(\mathrm{P}<0.001$, Table 1). In contrast, there was no obvious relationship between PPA1 expression levels and patient age, ascites or tumor distribution.

\section{Prognostic potential of PPA1 in OSC}

The 5-year overall survival of the cohort was $57.47 \%$ (Fig. 2A). No statistical correlation of survival with ascites or tumor distribution was found (Table 2). Univariate analysis revealed that age, pathological grade, LN metastasis and FIGO stage were all significantly correlated with the overall survival time (Table 2, Fig. 2B-E). Moreover, patients with low PPA1 expression showed better clinical outcome compared to those with high PPA1 expression $(\mathrm{P}=$ 0.020, Fig. 2F).

According to the results of univariate analysis, age, pathological grade, LN metastasis, FIGO stage and PPA1 expression were included in further multivariate analysis (Table 3). Among them, LN metastasis was an independent prognostic factor for overall survival $(\mathrm{HR}=1.88,95 \% \mathrm{CI}$ 1.23- 2.39, $\mathrm{P}=0.032$ ). FIGO stage was another significant factor with the hazard ratio as $2.14(95 \%$ CI $1.13-4.04, \mathrm{P}=$ 0.019). Moreover, our results indicated that PPA1 expression can also serve as a potential biomarker for predicting patients' post-operative survival ( $\mathrm{HR}=1.69,95 \%$ CI 1.08 $3.47, \mathrm{P}=0.046$ ).

PPA1 decreases the expression level of $p 53$ and increases $\beta$-catenin level

To explore how PPA1 participates in the OSC progression, we analyzed the expression patterns of PPA1 in different cell lines, including A2780 and OVCAR3 ovarian cancer cells and OSE normal ovarian epithelial cells. Western blot results demonstrated that PPA1 was expressed in normal ovarian epithelial cells, but its expression levels were higher in ovarian cancer cell lines (Fig. 3A). OVCAR3 cells showed higher PPA1 protein level compared with A2780 cells. Consequently, we overexpressed PPA1 in 

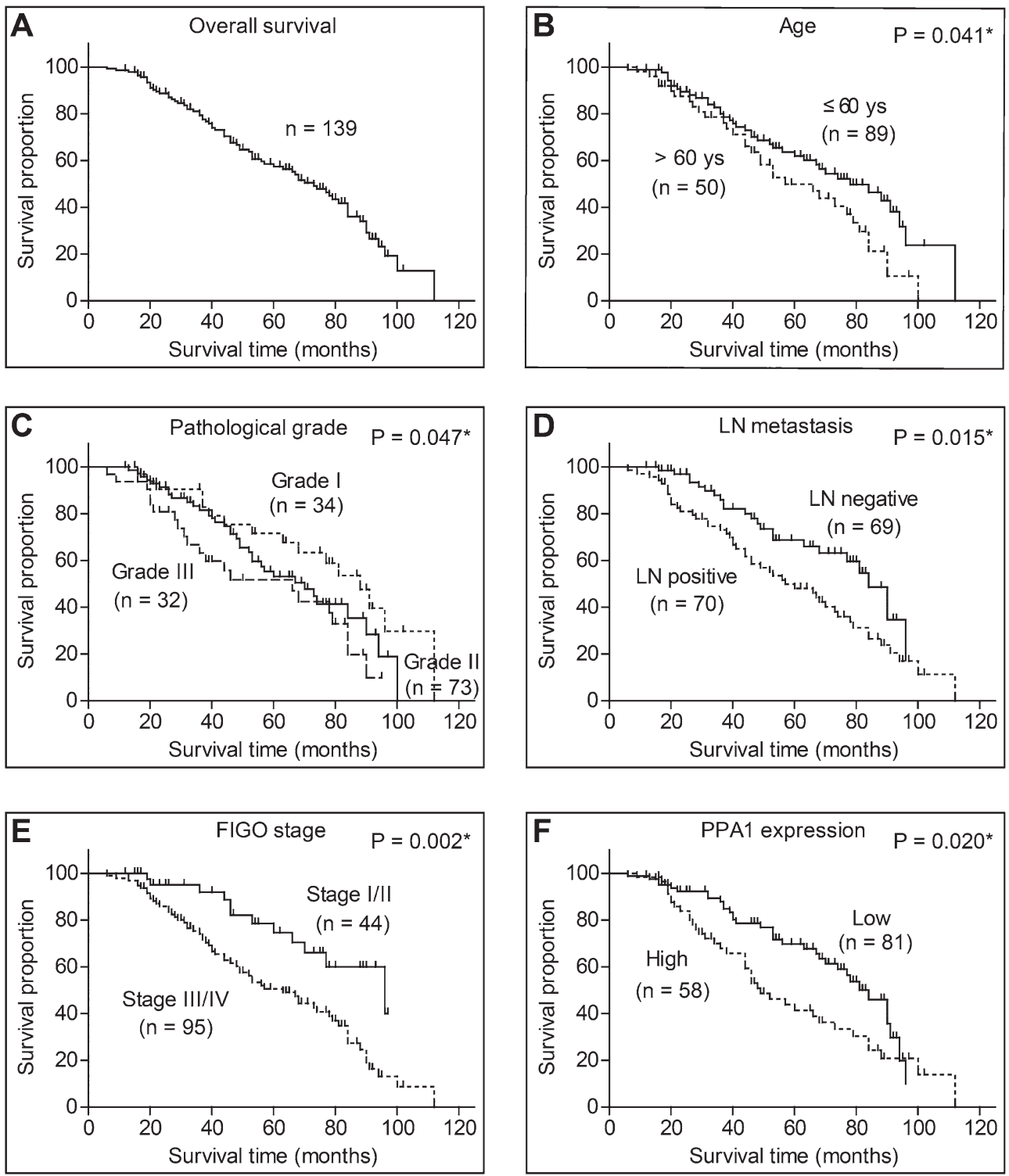

Fig. 2. Kaplan-Meier analysis of overall survival.

The 5-year overall survival of all enrolled patients was $57.47 \%$ (A). Patients with older age (B), poor pathological differentiation (C), positive LN metastasis (D), advanced FIGO stage (E) and higher PPA1 protein expression level (F) showed unfavorable overall survival (log-rank test, $\mathrm{P}<0.05)$.

A2780 cells and conversely knock-downed PPA1 expression in OVCAR3 cells to investigate the changes in the expression levels of cancer-related biomarkers, including p53, the tumor suppressor (Fig. 3B). We thus found that the p53 level was decreased by PPA1 overexpression in A2780 cells, while its expression level was increased in OVCAR3 cells upon PPA1 knockdown by siRNA (Fig. 3B). Moreover, PPA1 overexpression in A2780 cells caused the increase in the expression level of $\beta$-catenin, an important transcription factor that could promote tumor development. By contrast, PPA1 knockdown decreased the $\beta$-catenin level in OVCAR3 cells. Thus, PPA1 expression correlates with the $\beta$-catenin level.
PPA1 regulates the proliferation and invasion process of ovarian cancer cells

We also performed biological experiments to study the changes of tumorous characteristics upon PPA1 overexpression and silencing. MTT results showed that PPA1 overexpression positively regulated the viability of A2780 cells (Fig. 3C); in contrast, knockdown of PPA1 expression impaired the cell proliferation ability of OVCAR3 cells (Fig. 3D). Similarly, cell migration and invasion capacities were also regulated by PPA1, as shown by Matrigel Transwell assays (Fig. 3E, F).

\section{Discussion}

Lack of specific symptoms of EOC in its early stages is a significant factor contributing to its high mortality rate, 
Table 2. Overall survival analysis of OSC patients.

\begin{tabular}{lcccc}
\hline Variables & Cases & OS (months) & 5-year OS & P value \\
\cline { 3 - 3 } & & & & \\
\hline Age (years) & & & & $0.041^{*}$ \\
$\leq 60$ & 89 & $72.63 \pm 4.19$ & $61.9 \%$ & \\
$>60$ & 50 & $60.35 \pm 4.31$ & $49.9 \%$ & \\
Ascites (mL) & & & & 0.442 \\
None or $\leq 1000$ & 77 & $69.40 \pm 4.20$ & $57.2 \%$ & \\
$>1000$ & 62 & $64.40 \pm 4.13$ & $57.4 \%$ & \\
Distribution & & & & 0.958 \\
Unilateral & 103 & $67.82 \pm 3.51$ & $56.7 \%$ & \\
Bilateral & 36 & $64.58 \pm 5.61$ & $59.7 \%$ & \\
Pathological grade & & & & $0.047^{*}$ \\
G1 & 34 & $78.23 \pm 6.39$ & $71.6 \%$ & \\
G2 & 73 & $66.12 \pm 3.84$ & $53.1 \%$ & \\
G3 & 32 & $56.74 \pm 5.46$ & $51.7 \%$ & \\
LN metastasis & & & & $0.015^{*}$ \\
Negative & 69 & $73.20 \pm 3.77$ & $68.7 \%$ & \\
Positive & 70 & $60.86 \pm 4.13$ & $48.1 \%$ & \\
FIGO stage & & & & $0.002^{*}$ \\
I II & 44 & $79.27 \pm 4.36$ & $74.6 \%$ & \\
III IV & 95 & $61.73 \pm 3.50$ & $50.5 \%$ & \\
PPA1 expression & & & & \\
Low & 81 & $71.85 \pm 3.31$ & $69.7 \%$ & \\
High & 58 & $59.22 \pm 4.74$ & $41.4 \%$ & \\
\hline & & & & \\
\hline
\end{tabular}

OS, overall survival; LN, lymph node; FIGO, International Federation of Gynecology and Obstetrics.

Table 3. Multivariate analysis for overall survival of OSC patients.

\begin{tabular}{llll}
\hline Variables & HR & $\mathbf{9 5 \%}$ CI & P value \\
\hline $\begin{array}{l}\text { Age } \\
(>60 \text { vs. } \leq 60 \text { ys) }\end{array}$ & 1.23 & $0.71-2.12$ & 0.456 \\
$\begin{array}{l}\text { Pathological grade } \\
\text { (G3 vs. G1/G2) }\end{array}$ & 1.44 & $0.88-2.36$ & 0.147 \\
$\begin{array}{l}\text { LN metastasis } \\
\text { (Positive vs. Negative) }\end{array}$ & 1.88 & $1.23-2.39$ & $0.032^{*}$ \\
$\begin{array}{l}\text { FIGO stage } \\
\text { (III/IV vs. I/II) }\end{array}$ & 2.14 & $1.13-4.04$ & $0.019^{*}$ \\
$\begin{array}{l}\text { PPA1 expression } \\
\text { (High vs. Low) }\end{array}$ & 1.69 & $1.08-3.47$ & $0.046^{*}$ \\
\hline
\end{tabular}

HR, harzard ratio; 95\% CI, 95\% confidence interval; OS, overall survival; LN, lymph node; FIGO, International Federation of Gynecology and Obstetrics.

and over half of all deaths in women are caused by EOC (Barnholtz-Sloan et al. 2003). Despite significant diagnostic and surgical advances, and development of chemotherapeutic regimens, the clinical outcomes of EOC are still unsatisfied. Even the pathogenesis of EOC are unclear though numerous studies have been performed. Since recurrence and metastasis are the main hurdles for EOC treatment, post-operative follow-up and careful nursing can benefit patients' survival (Hay et al. 2016). Moreover, the requirement for more effective therapeutic strategies highlights the need to better understand the molecular mecha- nisms of EOC. Taking into consideration that energy dysregulation is critical in cancer progression, here we investigated the clinical significance and possible role of PPA1, an organic pyrophosphatase, in OSC.

The alteration of energy metabolism in tumor cells was firstly reported in 1960 s, which showed higher oxidative phosphorylation and energy consumption in tumor cells (Warburg 1955, 1958). Recently, researchers demonstrated that the hydrolysis of inorganic pyrophosphate is a highly exergonic reaction, thus serving as an alternative pathway for energy production in cells (Yi et al. 2012). Sub- 

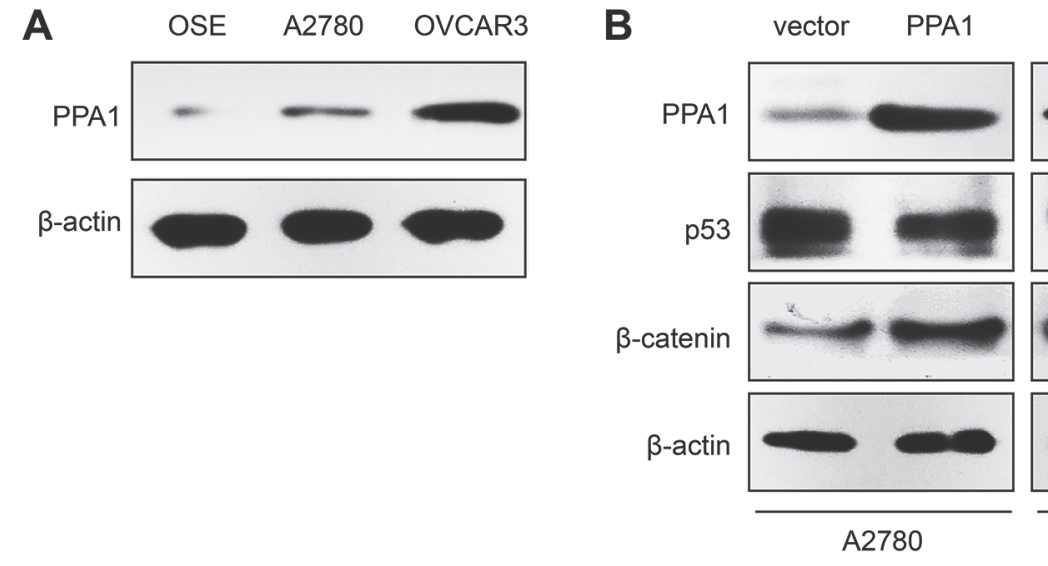

ncRNA SiRNA
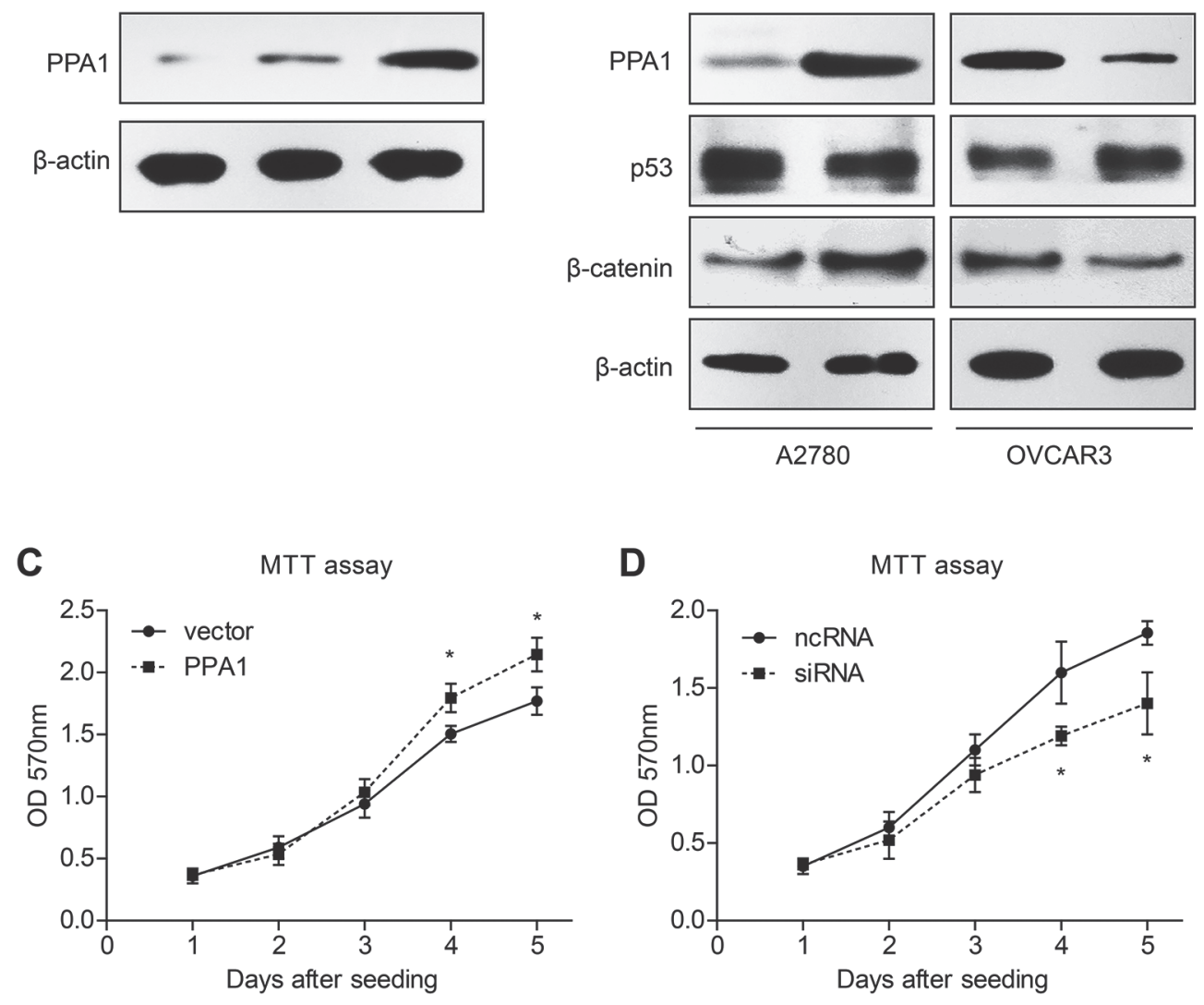

D MTT assay
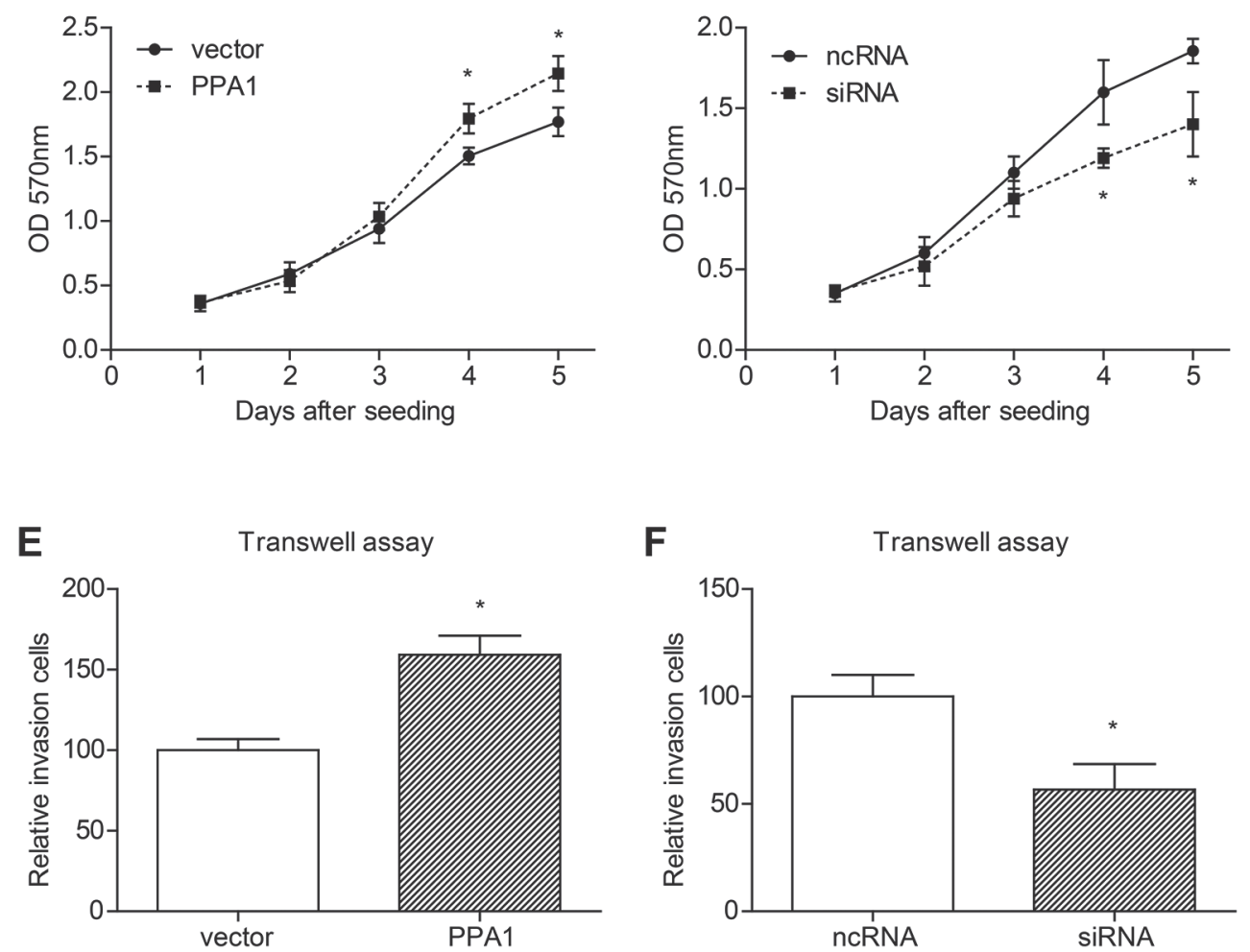

Fig. 3. Knockdown of PPA1 expression inhibits the proliferation and invasion of ovarian cancer cells.

(A) Western blot analysis, showing that the expression level of PPA1 is higher in ovarian cancer cell lines (A2780 and OVCAR3) than that in normal ovarian epithelial cells. (B) Overexpression of PPA1 was performed in A2780 cells, resulting in the decrease in the expression levels of p53 and the increase in the $\beta$-catenin levels (left panel). Conversely, PPA1 siRNA could successfully knockdown the expression of PPA 1 protein by $70 \%$ in OVCAR 3 cells (right panel), which was associated with the increased $\mathrm{p} 53$ level and the decreased $\beta$-catenin level. Each experiment was repeated for three times. (C) PPA1-overexpression could promote the proliferation of A2780 cells demonstrated by MTT assay. (D) PPA1 silencing down-regulated the cell viability in OVCAR3 cells (normalized with total cell number). Matrigel Transwell assay showed that the invasion capacity was enhanced in A2780 cells overexpressing PPA1 (E), but it was decreased in OVCAR3 cells transfected with PPA1 siRNA (F). Data are presented as the average fold change from triplicate experiments $(\mathrm{P}<0.05)$.

sequently, several groups reported the up-regulated expression of PPA1 in various solid tumors, including hepatocellular carcinoma (Megger et al. 2013), breast cancer (Mishra et al. 2015), and laryngeal squamous cell carcinoma (Bodnar et al. 2016). The PPA1 expression was also correlated with reduced overall survival of patients with gastric cancer (Yang et al. 2015). Moreover, PPA1 expression was reported to be associated with pathological grade in ovarian cancer (Luo et al. 2016), indicating that PPA1 may play an important role in carcinogenesis and development. However, the value of PPA1 in predicting clinical outcomes of OSC patients has not been in systematically investigated, 
which would be more significant in helping clinical prediction.

In the present study, we firstly verified PPA1 as an independent biomarker for poor prognosis of OSC patients. Moreover, PPA1 expression was significantly lower in the early stage group than that in the advanced stage group according to our results, which was inconsistent with the results by Luo and coworkers (Luo et al. 2016). In addition, we identified that PPA1 expression was correlated with positive LN metastasis, revealing the potential role of PPA1 for promoting OSC progression.

These clinical results prompted us to further investigate the possible mechanisms of PPA1 in regulating OSC development. Consistent with previous studies (Luo et al. 2016), our results also verified the role of PPA1 in regulating p53 levels. Since we identified the effects of PPA1 in promoting tumor cell proliferation and invasion, we next tested whether PPA1 can modulate the epithelial-mesenchymal transition (EMT) process. Beta-catenin is a critical transcription factor which has been extensively studied in malignancies (Rosenbluh et al. 2014; Monga 2015). Importantly, we demonstrated that the expression of $\beta$-catenin was positively regulated by PPA 1 levels. Although the signaling pathways between PPA1 and $\beta$-catenin need to be further illuminated by more basic experiments, our results provided evidence for the PPA1 as a potential chemotherapy target in the future.

In conclusion, high PPA1 protein expression level indicates poor prognosis of ovarian cancer patients, probably through regulating $\mathrm{p} 53$ and $\beta$-catenin pathways.

\section{Conflict of Interest}

The authors declare no conflict of interest.

\section{References}

Barnholtz-Sloan, J.S., Schwartz, A.G., Qureshi, F., Jacques, S., Malone, J. \& Munkarah, A.R. (2003) Ovarian cancer: changes in patterns at diagnosis and relative survival over the last three decades. Am. J. Obstet. Gynecol., 189, 1120-1127.

Bodnar, M., Luczak, M., Bednarek, K., Szylberg, L., Marszalek, A., Grenman, R., Szyfter, K., Jarmuz-Szymczak, M. \& Giefing, M. (2016) Proteomic profiling identifies the inorganic pyrophosphatase (PPA1) protein as a potential biomarker of metastasis in laryngeal squamous cell carcinoma. Amino Acids, 48, 1469-1476.

Chen, G., Gharib, T.G., Huang, C.C., Thomas, D.G., Shedden, K.A., Taylor, J.M., Kardia, S.L., Misek, D.E., Giordano, T.J., Iannettoni, M.D., Orringer, M.B., Hanash, S.M. \& Beer, D.G. (2002) Proteomic analysis of lung adenocarcinoma: identification of a highly expressed set of proteins in tumors. Clin. Cancer Res., 8, 2298-2305.

Chen, Z., Gao, P. \& Li, Z. (2016) Expression of G Protein-coupled Receptor 56 Is an Unfavorable Prognostic Factor in Osteosarcoma Patients. Tohoku J. Exp. Med., 239, 203-211.

Cheon, D.J., Tong, Y., Sim, M.S., Dering, J., Berel, D., Cui, X., Lester, J., Beach, J.A., Tighiouart, M., Walts, A.E., Karlan, B.Y. \& Orsulic, S. (2014) A collagen-remodeling gene signature regulated by TGF-beta signaling is associated with metastasis and poor survival in serous ovarian cancer. Clin. Cancer Res., 20, 711-723.
Cho, K.R. \& Shih Ie, M. (2009) Ovarian cancer. Annu. Rev, Phytopathol., 4, 287-313.

Guo, S., Liu, H.D., Liu, Y.F., Liu, L., Sun, Q. \& Cui, X.J. (2015) Hepatoma-derived growth factor: a novel prognostic biomarker in intrahepatic cholangiocarcinoma. Tumour Biol., 36, 353-364.

Hay, C.M., Courtney-Brooks, M., Lefkowits, C., Hagan, T.L., Edwards, R.P. \& Donovan, H.S. (2016) Symptom management in women with recurrent ovarian cancer: Do patients and clinicians agree on what symptoms are most important? Gynecol. Oncol., 143, 367-370.

Jayson, G.C., Kohn, E.C., Kitchener, H.C. \& Ledermann, J.A. (2014) Ovarian cancer. Lancet, 384, 1376-1388.

Jung, J.U., Ravi, S., Lee, D.W., McFadden, K., Kamradt, M.L., Toussaint, L.G. \& Sitcheran, R. (2016) NIK/MAP3K14 Regulates Mitochondrial Dynamics and Trafficking to Promote Cell Invasion. Curr. Biol., 26, 3288-3302.

Liu, H., Zhang, Q., Li, K., Gong, Z., Liu, Z., Xu, Y., Swaney, M.H., Xiao, K. \& Chen, Y. (2016) Prognostic significance of USP33 in advanced colorectal cancer patients: new insights into betaarrestin-dependent ERK signaling. Oncotarget, 13219.

Luo, D., Wang, G., Shen, W., Zhao, S., Zhou, W., Wan, L., Yuan, L., Yang, S. \& Xiang, R. (2016) Clinical significance and functional validation of PPA1 in various tumors. Cancer med., 5, 2800-2812.

Megger, D.A., Bracht, T., Kohl, M., Ahrens, M., Naboulsi, W., Weber, F., Hoffmann, A.C., Stephan, C., Kuhlmann, K., Eisenacher, M., Schlaak, J.F., Baba, H.A., Meyer, H.E. \& Sitek, B. (2013) Proteomic differences between hepatocellular carcinoma and nontumorous liver tissue investigated by a combined gel-based and label-free quantitative proteomics study. Mol. Cell Proteomics, 12, 2006-2020.

Mishra, D.R., Chaudhary, S., Krishna, B.M. \& Mishra, S.K. (2015) Identification of Critical Elements for Regulation of Inorganic Pyrophosphatase (PPA1) in MCF7 Breast Cancer Cells. PLoS One, 10, e0124864.

Monga, S.P. (2015) beta-Catenin Signaling and Roles in Liver Homeostasis, Injury, and Tumorigenesis. Gastroenterology, 148, 1294-1310.

Pan, C., Liu, H.D., Gong, Z., Yu, X., Hou, X.B., Xie, D.D., Zhu, X.B., Li, H.W., Tang, J.Y., Xu, Y.F., Yu, J.Q., Zhang, L.Y., Fang, H., Xiao, K.H., Chen, Y.G., et al. (2013) Cadmium is a potent inhibitor of PPM phosphatases and targets the M1 binding site. Sci. Rep., 3, 2333.

Rajadurai, C.V. \& Havrylov, S. (2016) 5'-Inositol phosphatase SHIP2 recruits Mena to stabilize invadopodia for cancer cell invasion. J. Cell Biol., 214, 719-734.

Rosenbluh, J., Wang, X. \& Hahn, W.C. (2014) Genomic insights into WNT/beta-catenin signaling. Trends Pharmacol. Sci., 35, 103-109.

Siegel, R.L., Miller, K.D. \& Jemal, A. (2016) Cancer statistics, 2016. CA Cancer J. Clin., 66, 7-30.

Signoretti, S., Di Marcotullio, L., Richardson, A., Ramaswamy, S., Isaac, B., Rue, M., Monti, F., Loda, M. \& Pagano, M. (2016) Oncogenic role of the ubiquitin ligase subunit Skp2 in human breast cancer. J. Clin. Invest., 126, 4387.

Terry, K.L., Schock, H., Fortner, R.T., Husing, A., Fichorova, R.N., Yamamoto, H.S., Vitonis, A.F., Johnson, T., Overvad, K., Tjonneland, A., Boutron-Ruault, M.C., Mesrine, S., Severi, G., Dossus, L., Rinaldi, S., et al. (2016) A Prospective Evaluation of Early Detection Biomarkers for Ovarian Cancer in the European EPIC Cohort. Clin. Cancer Res., 22, 4664-4675.

Tomonaga, T., Matsushita, K., Yamaguchi, S., Oh-Ishi, M., Kodera, Y., Maeda, T., Shimada, H., Ochiai, T. \& Nomura, F. (2004) Identification of altered protein expression and post-translational modifications in primary colorectal cancer by using agarose two-dimensional gel electrophoresis. Clin. Cancer Res., 10, 2007-2014.

Vang, R., Shih Ie, M. \& Kurman, R.J. (2009) Ovarian low-grade 
and high-grade serous carcinoma: pathogenesis, clinicopathologic and molecular biologic features, and diagnostic problems. Adv. Anat. Pathol., 16, 267-282.

Warburg, O. (1955) [Measurement of energy metabolism in photosynthesis by means of a large-surface bolometer]. Biochim. Biophys. Acta, 18, 163-164.

Warburg, O. (1958) [The effect of hydrogen peroxide on cancer cells and on embryonic cells]. Acta Unio Int. Contra Cancrum, 14, 55-57.
Yang, Y., Cai, J., Yin, J., Wang, D., Bai, Z., Zhang, J., Wang, K., Yu, G. \& Zhang, Z. (2015) Inorganic pyrophosphatase (PPA1) is a negative prognostic marker for human gastric cancer. Int. J. Clin. Exp. Pathol., 8, 12482-12490.

Yi, Y.J., Sutovsky, M., Kennedy, C. \& Sutovsky, P. (2012) Identification of the inorganic pyrophosphate metabolizing, ATP substituting pathway in mammalian spermatozoa. PLoS One, 7, e34524. 